\title{
Differences in fibrin fiber diameters in healthy individuals and thromboembolic ischemic stroke patients
}

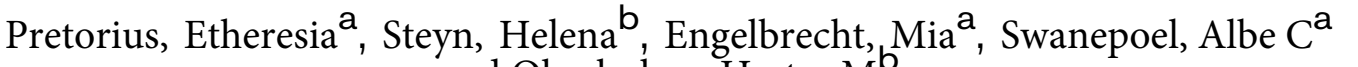 \\ and Oberholzer, Hester $\mathrm{M}^{\mathrm{b}}$
}

\author{
aDepartment of Physiology \\ bDepartment of Anatomy, School of Medicine, Faculty of Health Sciences, University of Pretoria, South Africa \\ Correspondence to Etheresia Pretorius, Department of Physiology, Faculty of Health Sciences, University of Pretoria, \\ Private Bag x323, ARCADIA, 0007, South AfricaTel: +27 12420 2864; fax: +27 12319 2240; e-mail: \\ resia.pretorius@up.ac.za
}

Cerebrovascular disease is one of the leading causes of death and the cause of long-term adult disability. An important characteristic of thromboembolic ischemic stroke is a prothrombotic or hypercoagulable state and altered fibrin clot structure, whereas a resistance to fibrinolysis is also present. An expansive fibrin network is created when adding thrombin, and in stroke, the network appears thickened, netted and matted, compared with that of healthy individuals. Although this is clearly visible in micrographs of patients, there is a need to quantify the changes. The current study, therefore, investigates fibrin fiber diameters in stroke patients and compares it to healthy individuals. The fiber diameters were measured in nanometres, with University of Texas Health Science Center at San Antonio (UTHSCSA) Image Tool. A total of 100 measurements were done for each of the 12 patients in the healthy control group, and the same number of measurements was done for 12 stroke patients. These measurements were statistically analysed with NCSS 2007, using a significance level of 0.05 . Normality was assessed with the Shapiro-Wilk W test and the thickest and thinnest fiber of each individual in the two groups was quantified and differences between groups were assessed with the Student's $t$-test. Results showed that there is a statistical difference in fibrin fiber thickness during thromboembolic ischemic stroke. We conclude that the changed coagulation and hemostasis, typically associated with stroke, causes a statistically relevant change in fibrin thickness, and that this netted and matted network is more resistant to lyses.

\section{Introduction}

Cerebrovascular disease is the third leading cause of death in the United States [1] and stroke is the leading cause of long-term adult disability [2]. Worldwide, ischemic stroke accounts for approximately $85 \%$ of stroke cases [2]. Hypercoagulation and microthrombi formation, which lead to microvascular occlusion and reduction in blood flow, are important events associated with stroke [3-6]. Impaired fibrinolysis also play an important role and research has shown that in ischemic stroke patients, there are significantly longer euglobulin clot lysis times, as well as higher levels of plasminogen activator inhibitor-1 and tissue-type plasminogen activator antigen [7].

An important characteristic of thromboembolic ischemic stroke is a prothrombotic or hypercoagulable state and altered fibrin clot structure. Also, in cryptogenic stroke and other ischemic strokes, a resistance to fibrinolysis is present [8].

In 2010, Undas et al. [8] compared acute ischemic stroke to controls, and showed that acute ischemic stroke patients produced clots that had $30.5 \%$ less porous network, were less susceptible to fibrinolysis and that the clots were $20.5 \%$ more compact with a $17.1 \%$ higher clot mass. Also, there was an increased (by 10.2\%) overall fiber thickness with $8 \%$ shorter lag phase of fibrin formation. In 2011, Pretorius et al. [9] showed that in thromboembolic ischemic stroke, fibrin networks have a changed ultrastructure. Fibrin networks were created within $24 \mathrm{~h}$ of stroke, using citrated blood, after the addition of thrombin. An expansive fibrin network is created when adding thrombin, and in stroke, the network appears thickened, netted and matted, compared to that of healthy individuals. The question now arose whether the changes in fibrin fiber network can be quantified. The current study, therefore, investigates fibrin fiber diameters in stroke patients and compares it to healthy individuals.

\section{Materials and methods Samples}

In our healthy individual research pool, we have hundreds of micrographs of fibrin networks. Whole blood was collected from individuals who did not have any of the following exclusion criteria: cardiovascular disease, anticoagulant medication, smoking, hormonal contraception or hormone replacement therapy, asthma, diabetes and inflammatory diseases, as all these conditions are known to influence the ultrastructure of fibrin networks $[10,11]$. From this research pool, 12 individuals were 
chosen and they represent typical fibrin morphology seen in healthy individuals. In our ongoing study of thromboembolic ischemic stroke patients, we have analysed 25 individuals. They were diagnosed according to the Trial of Org 10172 in Acute Stroke Treatment criteria [9]. From this sample, micrographs of 12 patients were chosen for the current study. Initially, fibrin networks were prepared by collecting whole blood from the patients within $24 \mathrm{~h}$ after the onset of stroke. The blood was processed to form fibrin networks and micrographs were taken of the networks. The micrographs were methodically viewed to establish the consistency of the ultrastructure, and one representative micrograph of each individual was chosen for further analysis.

\section{Preparation of fibrin clots}

Blood was centrifuged at $227 \mathrm{rpm}$ for $2 \mathrm{~min}$ to obtain platelet-rich plasma (PRP). Thrombin (provided by the South African National Blood Services) was used to prepare fibrin clots. The thrombin is at a concentration of $20 \mathrm{U} / \mathrm{ml}$ and was prepared in a biological buffer containing $0.2 \%$ human serum albumin. When thrombin is added to PRP, fibrinogen is converted to fibrin and intracellular platelet components, for example, transforming growth factor, platelet-derived growth factor and fibroblastic growth factor are released into the coagulum. Ten microliter of human PRP was mixed with $10 \mu \mathrm{l}$ of human thrombin. The PRP and thrombin mix was immediately transferred with a pipette tip to a $0.2 \mu \mathrm{m}$ Millipore membrane to form the coagulum (fibrin clot) on the membrane. This Millipore membrane was then placed in a Petri dish on filter paper damped with phosphatebuffered saline (PBS; to create a humid environment) and kept at $37^{\circ} \mathrm{C}$ for $10 \mathrm{~min}$. This step was followed by a washing process in which the Millipore membranes with the coagula were placed in PBS and magnetically stirred for $20 \mathrm{~min}$. This step is necessary to remove any blood proteins trapped within the fibrin network.

\section{Preparation of washed fibrin clot for scanning electron microscopy}

Washed fibrin clots were fixed in 2.5\% gluteraldehyde in Dulbecco's PBS in a $0.075 \mathrm{~mol} / \mathrm{l}$ phosphate buffer at a $\mathrm{pH}$ of 7.4 for 1 hour. Each fibrin clot was rinsed thrice in phosphate buffer for $5 \mathrm{~min}$ before being fixed for $30 \mathrm{~min}$ with $1 \%$ osmium tetraoxide. The samples were again rinsed thrice with PBS for 5 min and were then dehydrated serially in 30,50,70,90\% ethanol and three times with $100 \%$ ethanol. The scanning electron microscopy (SEM) procedures were completed by critical point drying of the samples, mounting, coating with carbon and examination of the tissue with a ZEISS ULTRA and FEG scanning electron microscope.

\section{Statistical analysis}

After visualization of the fibrin networks using SEM, micrographs were taken at 40000 times machine magnification. A representative photograph of each sample was selected, a $10 \times 10$ grid was drawn onto the picture (grids shown in Fig. 1) and the picture was enlarged to a degree that the thinnest fibers could be measured easily. One fiber was randomly selected out of each block of the grid, to ensure that a fiber was not measured twice, and that fibers were systematically measured to prevent observer bias; this resulted in 100 measurements per individual photograph. The diameter was measured in nanometres, with UTHSCSA Image Tool version 3.00. A total of 1200 measurements were done for the healthy control group; the same number of measurements was done using micrographs of stroke patients. Measurements were repeated by two observers. These measurements were statistically analysed with NCSS 2007 version 07.1.20 (NCSS LLC, Kaysville, Utah, USA), using a significance level of 0.05 . Normality was assessed with the Shapiro-Wilk W test of the two groups, and frequency histograms were constructed to display the distribution of fiber diameters graphically. The thickest and thinnest fiber of each individual in the two groups was quantified, and made into four groups: maximum of the control group, minimum of the control
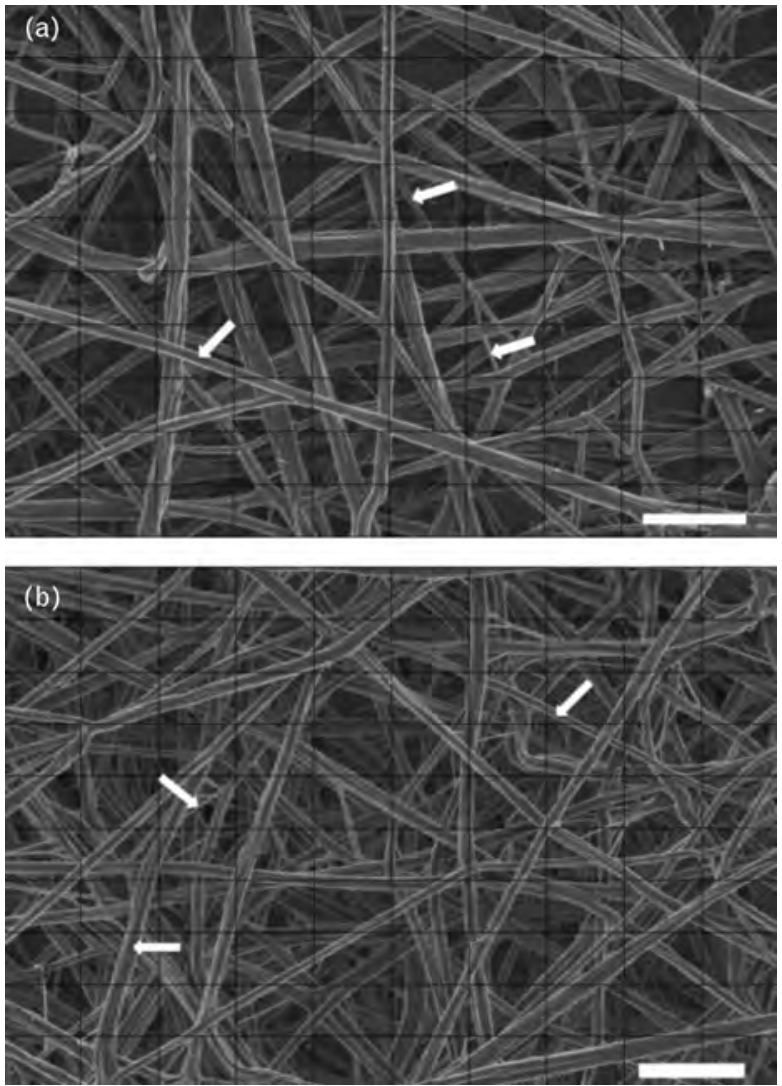

Fig. 1. Micrographs from two healthy individuals showing grids used to identify fibers for measurement. Arrows indicate examples of fibers that were measured. All scale bars $=1 \mu \mathrm{m}$. 
group, maximum of the stroke group and minimum of the stroke group. The difference between group first and third groups, as well as the difference between group second and fourth groups were assessed with the Student's $t$-test.

\section{Results}

Figure 1a and b show micrographs of individuals that are representative of the control group. Typically, fibrin networks of healthy individuals form thick, major fibers with fine minor fibers dispersed amongst the major fibers. Figure $2 \mathrm{a}-\mathrm{f}$ shows micrographs from the patient stroke group. As previously noted by Pretorius et al. [9], in ischemic stroke patients, fibrin fiber arrangement changes after the addition of thrombin to citrated blood, to form a matted and netted fibrin mass. As can be seen in Fig. 3 (which shows histograms of the frequency distributions of fiber diameters) the stroke patient group has a much larger amount of thin fiber counts than the control group, with very few thick major fibers.
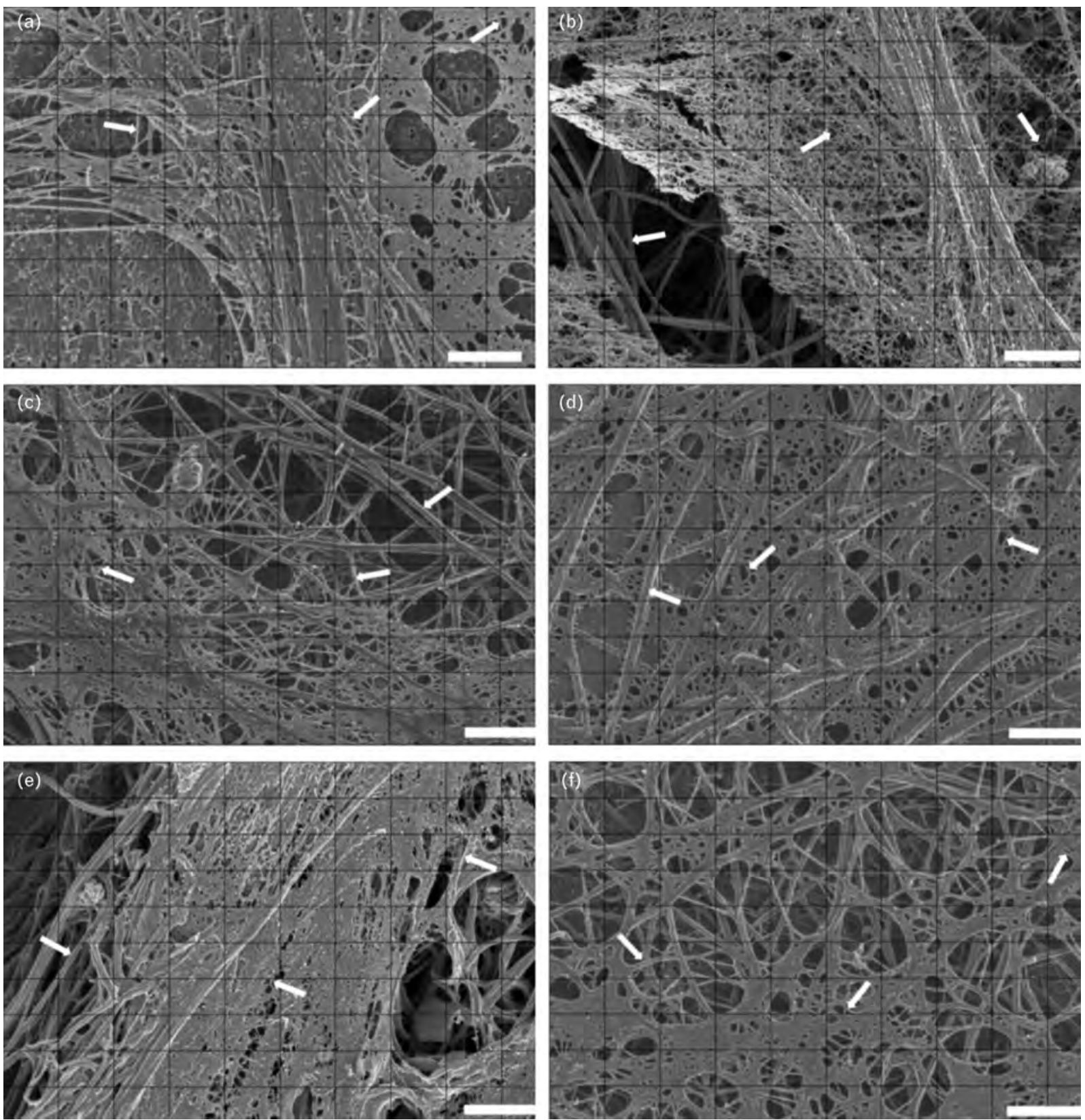

Fig. 2. Micrograph examples of six different thromboembolic stroke patients showing grids used to identify fibers for measurement. Arrows indicate examples of fibers that were measured. All scale bars $=1 \mu \mathrm{m}$. 
(a)

Histogram of control group

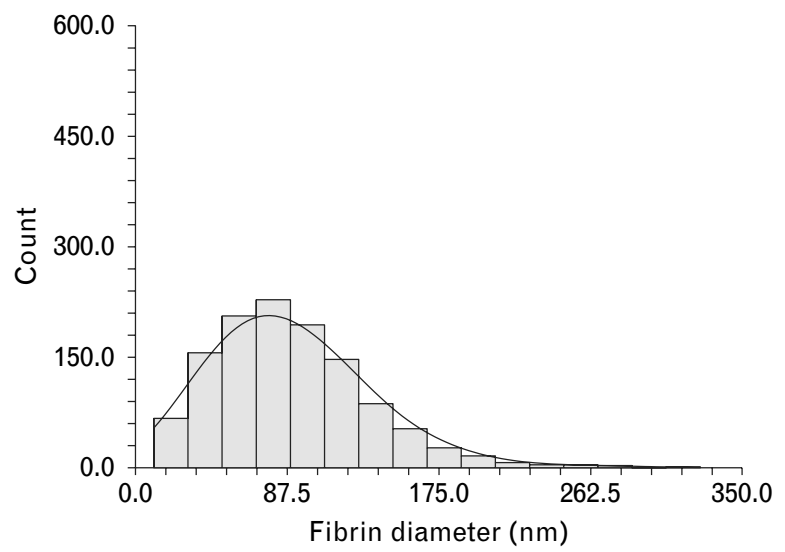

(b)

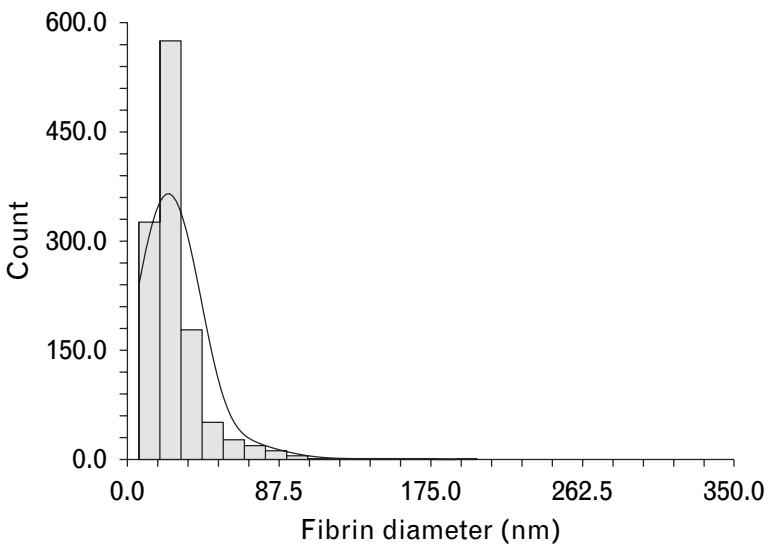

Fig. 3. Histograms illustrating the frequency distribution of (a) healthy individuals and (b) stroke patients' fibrin fiber diameters.

Table 1 shows the mean and SD of the overall measurements, and also of the maximum and minimum groups. In the current analysis, the Shapiro-Wilk W test was used to assess normality; both groups showed that the fibrin diameters were not normally distributed, in which both the control and stroke patient group had $P$-values of less than 0.000. It is expected that the groups will not show a normal distribution, as a fibrin clot consists of thick, major and thin minor fibers [12]. Frequency histograms were plotted in NCSS for each group to give a visual display of the distribution of fiber diameters (Fig. 3).

\section{Discussion}

Fibrin structure and stability and abnormal fibrin structure is a novel link between inflammation and thrombosis [13]. Previous research also suggested that fibrin clots have increased fiber diameter and density in stroke patients $[8,14]$. Also in their 2010 study, Undas et al. [14] suggested that scanning electron microscopy of fibrin clots could provide direct data on fibrin thickness and pore structure.

For the current analysis, micrographs of 12 healthy individuals and 12 stroke patients were chosen. The 12 control micrographs were chosen from a database consisting of thousands of control micrographs and these micrographs, therefore, represent typical morphology for

Table 1 Analysis of fibrin fiber diameters of thromboembolic stroke patients and healthy individuals

\begin{tabular}{lcc}
\hline & Control group & Stroke group \\
\hline Total mean \pm SD $(\mathrm{nm})$ & $89.87 \pm 44.16$ & $27.37 \pm 17.03$ \\
Maximum mean \pm SD $(\mathrm{nm})$ & $227.28 \pm 55.03$ & $95.54 \pm 47.43$ \\
Minimum mean \pm SD $(\mathrm{nm})$ & $23.69 \pm 9.48$ & $10.95 \pm 3.46$
\end{tabular}

controls. Currently, we have a micrograph database for 30 ischemic stroke patients. These micrographs also represent a typical morphology for ischemic stroke patients. A grid was overlaid over each micrograph, and one fiber measured per area grid-block (100 measurements per micrograph; Figs 1 and 2). This assured a nonbiased choosing of fibers, systematically over whole area of micrograph. The number of measurements from each of the two samples showed statistical significant results between the two groups. The distribution of both groups shows a 'merged' distribution pattern in which there are no two clear peaks showing the thick and thin fibers (Fig. 3); this suggests a possible intermediate thickness fiber because in a group in which there are only thick and thin fibers, a bimodal distribution pattern would be expected.

In the control group, the single largest measurement of each individual was taken and classified in a 'maximum' group, whereas the single smallest measurement of each individual was classified as a 'minimum' group. The same was done for the stroke patients, and significant difference was then determined using the Students' $t$-test between maximum control and stroke groups, and between minimum control and stroke groups. Table 1 shows the mean and SD of the overall measurements, and also of the maximum and minimum groups. Between group analyses of the maximum measurements resulted in a $P$-value of 0.000 , which suggest that there is a significant difference between the control and stroke patients' thickest fibers. Between group analyses of the minimum measurements resulted in a $P$-value of 0.002 , which suggest that there is a significant difference between the control and stroke patients' thinnest fibers. 
Currently, the use of morphology to investigate clots is debated in the literature. In an editorial in the journal Stroke in 2011, the authors discussed the increasing use of mechanical embolectomy devices [15]. This methodology allows for retrieving fresh clots from patients with acute stroke and successive morphological characterization. It, therefore, seems that visualization of clot structure in ischemic stroke might give us insights into the disease and possible treatment regimes [15]. The present research might add to the knowledge of clot structure and function and stimulate further research, as it shows that there is a statistical difference in fibrin fiber thickness during thromboembolic ischemic stroke. Previous research has suggested that in conditions that affect the pathophysiology, fibrin polymerizes differently, compared with the typical process present in healthy individuals [16]. Clots in healthy and diseased individuals may, therefore, differ in structure as well as physical and chemical properties; and these properties may change due to the internal biological environment of the individual. Here we show that in stroke, fibrin polymerization is changed and this caused a layered, matted fibrin clot structure. In 2010, Undas et al. [14] showed that there are unfavourably altered fibrin clot properties in stroke. Here we suggest that the changed coagulation and hemostasis typically associated with stroke, causes a statistically relevant change in fibrin thickness.

\section{Acknowledgements Conflicts of interest}

There are no conflicts of interest.

\section{References}

1 Marsh JD, Keyrouz SG. Stroke prevention and treatment. J Am Coll Cardiol 2010; 56:683-691.
2 Sandercock PA, Counsell C, Kamal AK. Anticoagulants for acute ischaemic stroke. Cochrane Database Syst Rev 2008; 4:CD000024.

3 Kawai H, Yuki S, Sugimoto J, Tamao Y. Effects of a thrombin inhibitor, argatroban, on ischemic brain damage in the rat distal middle cerebral artery occlusion model. J Pharmacol Exp Ther 1996; 278:780785.

4 Morris DC, Zhang L, Zhang ZG, Lu M, Berens KL, Brown PM, et al. Extension of the therapeutic window for recombinant tissue plasminogen activator with argatroban in a rat model of embolic stroke. Stroke 2001; 32:2635-2640.

5 Matijevic N, Wu KK. Hypercoagulable states and strokes. Curr Atheroscler Rep 2006; 8:324-329.

6 Park JS, Park SS, Koh EJ, Eun JP, Choi HY. Treatment for patients with acute ischemic stroke presenting beyond six hours of ischemic symptom onset: effectiveness of intravenous direct thrombin inhibitor, argatroban. $J$ Korean Neurosurg Soc 2010; 47:258-264.

7 Vucković BA, Djerić MJ, llić TA, Canak VB, Kojić-Damjanov SLj, Zarkov MG, Cabarkapa VS. Fibrinolytic parameters, lipid status and lipoprotein(a) in ischemic stroke patients. Srp Arh Celok Lek 2010; 138 (Suppl 1):1217.

8 Undas A, Podolec P, Zawilska K, Pieculewicz M, Jedliński I, Stępień E, et al. Altered fibrin clot structure/function in patients with cryptogenic ischemic stroke. Stroke 2009; 40:1499-1501.

9 Pretorius E, Swanepoel AC, Oberholzer HM, Van der Spuy WJ, Duim W, Wessels PF. A descriptive investigation of the ultrastructure of fibrin networks in thrombo-embolic ischemic stroke. J Thromb Thrombolysis 2011; 31:507-513.

10 Pretorius E, Oberholzer HM, Van der Spuy WJ, Meiring JH. The changed ultrastructure of fibrin networks during use of oral contraception and hormone replacement. J Thromb Thrombolysis 2010; 30:502506.

11 Pretorius E, Oberholzer HM, Van der Spuy WJ, Meiring JH. Smoking and coagulation: the sticky fibrin phenomenon. Ultrastruct Pathol 2010; 34:236-239.

12 Pretorius E, Vieira WA, Oberholzer HM, Auer REJ. Comparative scanning electron microscopy of platelets and fibrin networks of humans and different animals. Int J Morpho/ 2009; 27:69-76.

13 Campbell RA, Overmyer KA, Selzman CH, Sheriden BC, Wolberg AS. Contributions of extravascular and intravascular cells to fibrin network formation, structure, and stability. Blood 2009; 114:4886-4896.

14 Undas A, Slowik A, Wolkow P, Szczudlik A, Tracz W. Fibrin clot properties in acute ischemic stroke: relation to neurological deficit. Thromb Res 2010; 125:357-361.

15 Minnerup J, Kleinschnitz C. Visualization of clot composition in ischemic stroke: do we get what we see? Stroke $2011 ;$ 42:1193-1194.

16 Weisel JW. Structure of fibrin: impact on clot stability. J Thromb Haemost 2007; 1:116-124. 